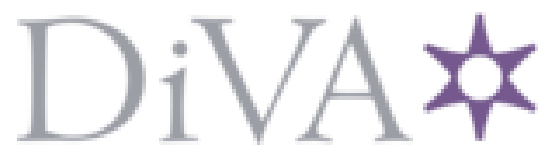

http://www.diva-portal.org

This is the published version of a paper published in Applied Physics Letters.

Citation for the original published paper (version of record):

Schwab, M B., Sävert, A., Jäckel, O., Polz, J., Schnell, M. et al. (2013)

Few-cycle optical probe-pulse for investigation of relativistic laser-plasma interactions.

Applied Physics Letters, 103(19): 191118

https://doi.org/10.1063/1.4829489

Access to the published version may require subscription.

N.B. When citing this work, cite the original published paper.

Permanent link to this version:

http://urn.kb.se/resolve?urn=urn:nbn:se:umu:diva- 134422 


\title{
Few-cycle optical probe-pulse for investigation of relativistic laser-plasma interactions
}

\author{
M. B. Schwab, ${ }^{1, a)}$ A. Sävert, ${ }^{1}$ O. Jäckel, ${ }^{1,2}$ J. Polz, ${ }^{1}$ M. Schnell, ${ }^{1}$ T. Rinck, ${ }^{1}$ L. Veisz, ${ }^{3}$ \\ M. Möller, ${ }^{1}$ P. Hansinger, ${ }^{1}$ G. G. Paulus, ${ }^{1,2}$ and M. C. Kaluza ${ }^{1,2}$ \\ ${ }^{1}$ Insitut für Optik und Quantenelektronik, Max-Wien-Platz 1, 07743 Jena, Germany \\ ${ }^{2}$ Helmholtz-Institut Jena, Fröbelstieg 3, 07743 Jena, Germany \\ ${ }^{3}$ Max-Planck-Institut für Quantenoptik, Hans-Kopfermann-Straße 1, 85748 Garching, Germany
}

(Received 17 June 2013; accepted 26 October 2013; published online 8 November 2013)

\begin{abstract}
The development of a few-cycle optical probe-pulse for the investigation of laser-plasma interactions driven by a Ti:sapphire, 30 Terawatt (TW) laser system is described. The probe is seeded by a fraction of the driving laser's energy and is spectrally broadened via self-phase modulation in a hollow core fiber filled with a rare gas, then temporally compressed to a few optical cycles via chirped mirrors. Shadowgrams of the laser-driven plasma wave created in relativistic electron acceleration experiments are presented with few-fs temporal resolution, which is shown to be independent of post-interaction spectral filtering of the probe-beam. (C) 2013 AIP Publishing LLC. [http://dx.doi.org/10.1063/1.4829489]
\end{abstract}

Research in the field of laser-particle acceleration relies heavily on the use of empirical data in order to verify complex simulations of laser-plasma interactions. ${ }^{1-4}$ Such data typically include, e.g., the accelerated particles' kinetic energy, their energy spread, the particle beam's divergence, and any secondary radiation emitted during the interaction. A deeper understanding of the data, simulations, and the phenomena they describe can be achieved by directly imaging the evolution of the plasma's density distribution using a pump-probe setup. Techniques such as shadowgraphy and interferometry have been regularly used to image density distributions in other fields of research. However, the temporal and spatial resolutions achieved are typically insufficient to observe the transient and fine-structured features present in underdense laser-plasma interactions. The investigation of these phenomena in the single-shot regime adds another level of complexity to these experiments. ${ }^{5-10}$

A well-known phenomenon on the femtosecondtimescale is described in Tajima and Dawson's discussion of the laser wakefield associated with a plasma wave generated in laser-electron acceleration experiments. ${ }^{11}$ The plasma wave is created via the ponderomotive force of a highintensity laser pulse as it propagates through underdense plasma. Using an electron density, $n_{e}$, around $10^{19} \mathrm{~cm}^{-3}$, the wavelength of the plasma wave can be calculated to be near $10 \mu \mathrm{m}$

$$
\lambda_{p}=\frac{2 \pi c}{\omega_{p}}=2 \pi c \sqrt{\frac{\varepsilon_{o} m_{e}}{n_{e} e^{2}}}
$$

Equation (1) shows the relationship in the nonrelativistic regime between the plasma wavelength $\lambda_{p}$, the speed of light $\mathrm{c}$, and the plasma frequency $\omega_{p}$, which is further defined with the electric permittivity $\varepsilon_{\mathrm{o}}$, electron mass $m_{e}$, and electron charge $e$. The electron density used in an experiment is usually chosen with regard to the laser's

\footnotetext{
${ }^{\text {a) }}$ Author to whom correspondence should be addressed. Electronic mail: matthew.schwab@uni-jena.de
}

parameters and the phenomenon being investigated. Furthermore, numerical simulations predict that the plasma wave may exhibit non-linear features such as wavefront curvature and breaking during the process of electron acceleration. A few-cycle, optical probe-pulse enables the direct observation of the plasma wave's fine structure, but demands a pulse duration that is well below what most of the current multi-TW class lasers with relativistic intensities can achieve.

The results presented in this paper primarily rely on the merging of two well-established laser technologies: a multiTW Ti:sapphire laser based on chirped pulse amplification (CPA), ${ }^{12}$ and a hollow core fiber (HCF) compressor system used to produce few-cycle pulses at optical frequencies. ${ }^{13-16}$ The combination of these two systems lends itself to new avenues of characterizing laser-plasma interactions in the single-shot regime with unprecedented resolution.

In this paper, we present the setup and describe the performance of a single-shot few-cycle probe system implemented on the Jena Ti:sapphire (JETI) $30 \mathrm{TW}$ laser at the Institut für Optik und Quantenelektronik in Jena, Germany. With this addition to the laser system, which may be easily implemented into existing TW-lasers, a deeper insight into laser-plasma interactions can be achieved.

There exists a large variety of pump-probe setups for the investigation of laser-plasma interactions. Due to the length of a TW laser chain and the resulting timing jitter between shots, the probe is typically split off from the pump as close to the interaction region as possible to ensure pulse synchronization. By minimizing the overall temporal dispersion of the probe due to subsequent propagation in air or glass, a probe with a pulse duration equal to, ${ }^{5}$ or only slightly longer than that of the pump can be achieved. ${ }^{8}$ Often the probe is used to transversely illuminate the laser-plasma interaction, relative to the pump's direction of propagation. Imaging methods such as shadowgraphy, interferometry, or polarimetry are used to produce a single image of the laser-plasma interaction per laser shot. The evolution of this interaction can be further investigated by varying the time delay 
between the pump and probe during subsequent laser shots assuming each produces a repeatable interaction.

Along with transverse probing, several longitudinal probing methods also exist. Frequency Domain Holography $(\text { FDH })^{9}$ and Frequency Domain Shadowgraphy (FDS) ${ }^{10}$ are two such techniques that have seen success in imaging structures in underdense plasmas. Both methods, however, create images that are averaged over the whole time period in which the pump and probe co-propagate through the plasma. This can blur out any phenomena that evolve inside the plasma structure and becomes a critical drawback for probing in the wave-breaking regime ${ }^{17}$ where temporally evolving structures associated with particle acceleration lose image fidelity due to this averaging. Transverse probing with a few-cycle probe significantly reduces this loss of fidelity, can be performed with a relatively simple setup, and does not require computational support to produce an image.

The generation of a few-cycle probe-pulse depends on a method to significantly broaden the probe's spectrum, and to tailor its spectral phase to be flat. A well-established approach for large spectral bandwidth generation with pulse energy in the $\mu \mathrm{J}$ to $\mathrm{mJ}$ range is self-phase modulation (SPM) in a noble-gas filled HCF. ${ }^{18-20}$ It has been shown that this nonlinear phenomenon can produce a spectrum wide enough to support pulse durations below $4 \mathrm{fs} .{ }^{16}$

The described probe-beam in the JETI laser system is shown in Fig. 1. Its energy is initially split from the pump beam by a 99:1 dielectric beam splitter (BS) positioned at a $45^{\circ}$ incidence angle inside the vacuum beam line. The probe exits the vacuum beam line through a fused silica window (VW). An apodized aperture (AA) then cuts a roughly $10 \mathrm{~mm} 1 / \mathrm{e}^{2}$ diameter beam out of the larger beam profile.

The probe-beam accumulates significant group delay dispersion (GDD) due to transmission through various optics in the beam path before being focused into the gas-filled HCF by a thin lens (L). In order to ensure effective SPM in the HCF, the pulses are temporally recompressed via several reflections from chirped mirrors (CMs). The magnitude of spectral broadening in the HCF can be altered via a variety of parameters. ${ }^{13}$ This system depends primarily on gas type and pressure to generate sufficient spectral broadening to support few-cycle pulses. After exiting the HCF, the pulses

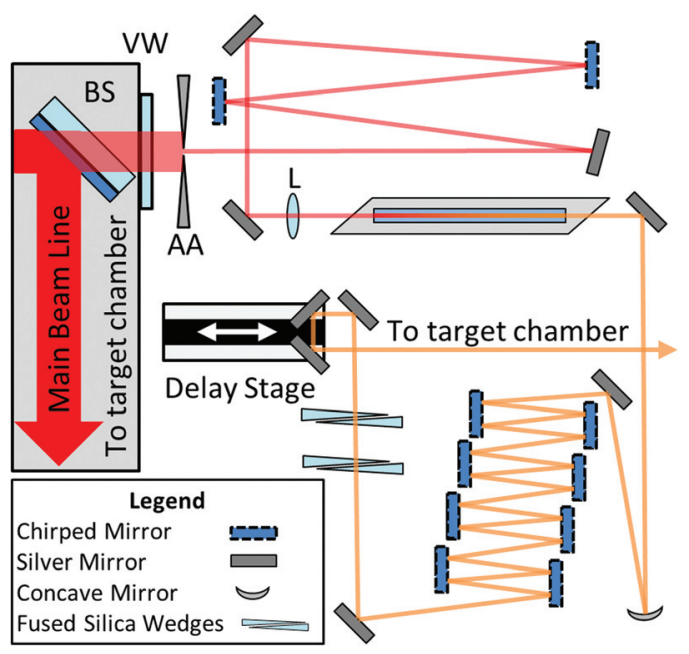

FIG. 1. Diagram of the few-cycle probe setup. are collimated and directed towards a group of eight CMs. This matched-set of CMs is designed to support $<7 \mathrm{fs}$ pulses in the wavelength range from 620 to $920 \mathrm{~nm}$. A motorized delay stage allows the relative timing between the pumpand probe-pulses to be varied within a $2 \mathrm{~ns}$ temporal window with a step size of $33 \mathrm{fs}$. After the probe exits the delay stage, it passes through two pairs of fused silica wedges. By driving the wedges together or apart their net GDD contribution can be varied allowing for fine compensation of the negative GDD created by the $8 \mathrm{CM}$ group.

The system was characterized using argon gas inside the HCF. The output pulse energy from the HCF was measured to be $300 \pm 15 \mu \mathrm{J}$, giving a transmission efficiency of approximately $60 \%$ for input pulse energies of $500 \pm 25 \mu \mathrm{J}$. Argon gas showed sufficient spectral broadening with 0.4 bar of pressure to support Fourier-limited pulses of $4.4 \mathrm{fs}$ full width at half maximum (FWHM). The average FWHM pulse duration including one standard deviation was measured to be $5.9 \pm 0.4$ fs (Fig. 2) using an FC-SPIDER from APE $\mathrm{GmbH}$ in single-shot mode.

The primary purpose of the probe-beam described above was to act as a back-lighting probe in a Laser Wakefield Acceleration (LWFA) experiment. Fig. 3 shows the experimental setup. The JETI pump-beam was focused into a hydrogen gas jet by an F/13 off-axis parabola. The supersonic gas nozzle had a circular opening $2.4 \mathrm{~mm}$ in diameter and produced a top-hat-like density profile. The backing pressure was varied to produce a plasma density in the central region of the top-hat near $1.5 \times 10^{19} \mathrm{~cm}^{-3}$ at a distance of $1.25 \mathrm{~mm}$ above the nozzle's opening. This density assumes full ionization of the $\mathrm{H}_{2}$ gas used. Measurement of the neutral gas density distribution above the nozzle was previously performed using tomographic reconstruction methods. ${ }^{21}$

The probe-beam was directed transversally through the laser-plasma interaction in a side-view setup. Gradients in the plasma's refractive index cause the probe-beam to refract from its collimated path.
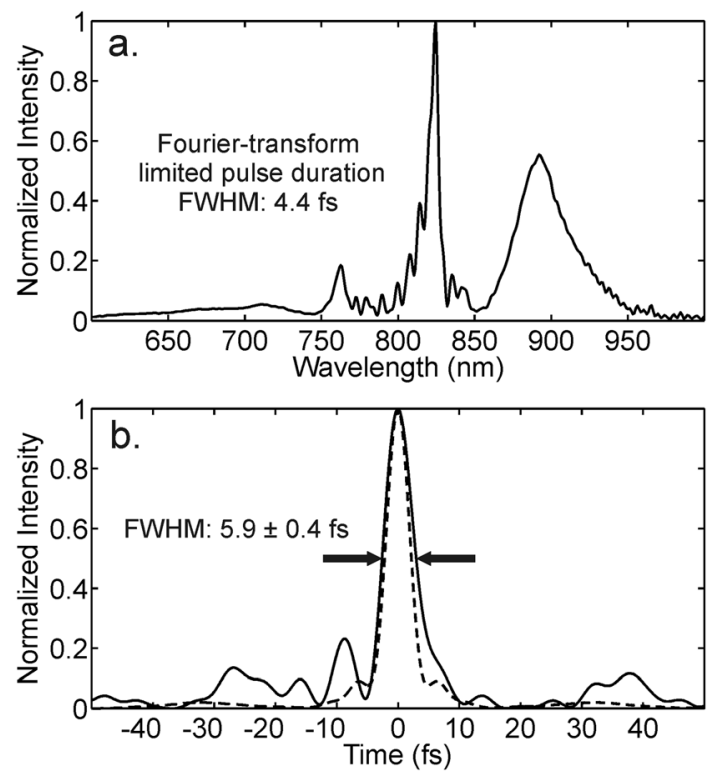

FIG. 2. Measurements from FC Spider (APE GmbH). (a) Spectral intensity. (b) Temporal intensity. Fourier-limited pulse duration (dashed) and measured temporal profile (solid). 


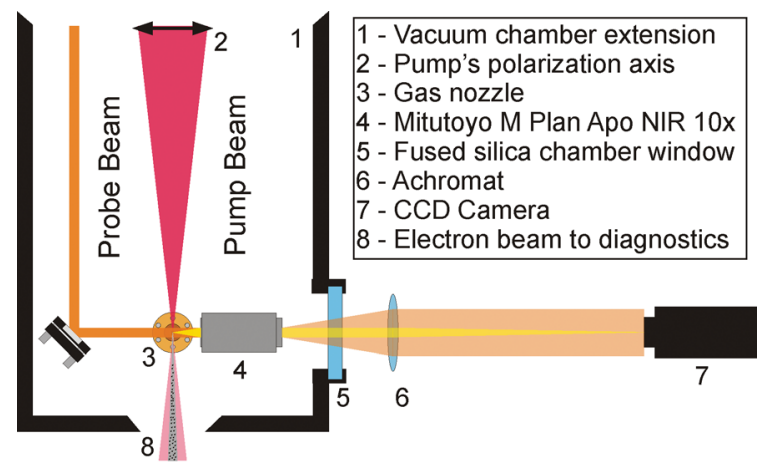

FIG. 3. Shadowgraphic imaging system.

$$
\eta=\sqrt{1-\frac{n_{e}}{n_{c}}}=\sqrt{1-\frac{n_{e} e^{2}}{\omega_{L}^{2} \gamma m_{e} \varepsilon_{o}}} .
$$

Equation (2) describes the refractive index of the plasma, $\eta$, as a function of the local electron density, $\mathrm{n}_{\mathrm{e}}$, inside the plasma with $\mathrm{n}_{\mathrm{c}}$ denoting the critical density of the plasma for a given laser frequency, $\omega_{\mathrm{L}}$. Here, $\gamma$ the relativistic gamma factor takes into account the potentially relativistic nature of the electrons.

Probe-beam light refracting from any gradient of the plasma's refractive index was collected by a Mitutoyo M Plan Apo NIR $10 \times$ microscope objective $(\mathrm{NA}=0.26)$ and imaged onto a CCD array via an achromatic lens. The remaining unscattered probe-beam collected by the optical system contributed to the background intensity of the image. The resulting shadowgrams were recorded with a $0.5 \mu \mathrm{m} /$ pixel length scale.

Scattered light from the pump pulse propagating through the plasma can hinder imaging of the plasma wave, but is mitigated using two methods. First, the pump is polarized parallel to the shadowgraphy's imaging axis (see double-arrow in Fig. 3.), so that polarized scattering towards the imaging setup is suppressed. This orientation of the pump pulse's polarization is well suited for side-view optical probing, while the imaging channel in the top-view direction (not shown here) can be utilized to investigate scattered pump light. Second, the gas backing pressure used in the experiment was set low enough to avoid multiple self-injection points in the plasma wave and has the added benefit of further minimizing scattered light in the shadowgraphic imaging channel.

Fig. 4(a) shows a single-shot shadowgram of a lasergenerated plasma wave recorded using the few-cycle pulse as a transverse optical probe. The image shows the plasma wave's wavefront curvature and substructures. Here, the driving laser pulse propagates from left to right. No spectral filtering of the probe-beam was performed. This plasma wave was created during an interaction triggered by focusing a JETI laser pulse of approximately $750 \mathrm{~mJ}$ energy and $35 \mathrm{fs}$ FWHM duration to a vacuum peak intensity near $6.3 \times 10^{18} \mathrm{~W} / \mathrm{cm}^{2}$ into ionized hydrogen gas with a background electron density, $n_{e}$, of $\sim 1.5 \times 10^{19} \mathrm{~cm}^{-3}$. The periodic modulation of the plasma wave's electron density is clearly visible and resembles that seen in simulations. ${ }^{17}$ The plasma wavelength is measured to be $9.0 \pm 0.5 \mu \mathrm{m}$. A line-out of the plasma wave (Fig. 4(b)) shows its on-axis normalized intensity modulation.

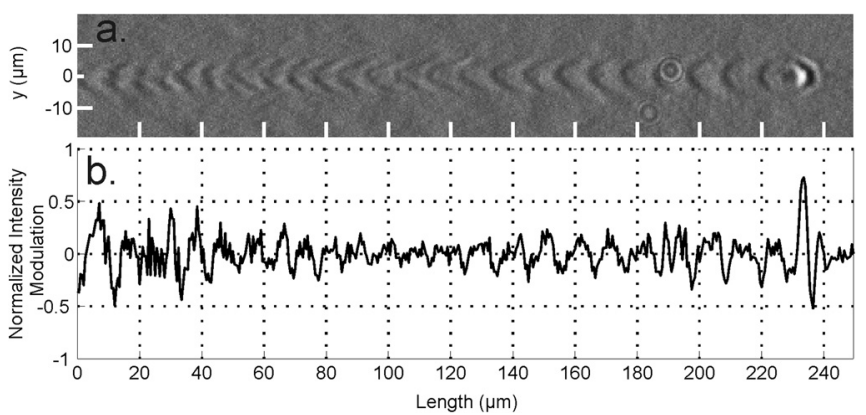

FIG. 4. (a) Shadowgram of a plasma wave propagating from left to right. Inhomogeneous background illumination has been subtracted and contrast has been enhanced. Diffraction patterns between 180 and $200 \mu$ m originate from dust particles. (b) On-axis line-out of modulation normalized to background (no contrast enhancement).

Clear substructures in the plasma wave including a variation of the wavefront curvature due to relativistic effects, a variation of the modulation strength along the wave and also slight variations of the plasma wavelength can be detected with this single-shot transverse probe-beam setup. Furthermore, the first period of the plasma wave (i.e., the one on the right end of the structure) shows a different substructure as compared to the trailing oscillations. This modified shape is most likely due to the position of the pumppulse's peak intensity as it propagates through the plasma and forms the plasma wake. While this image already shows more fine-structure of the plasma wave than previously seen in experiments, further investigations of the laser-plasma interaction using this probe setup hold the promise to gain deeper insight into the evolution of the plasma wave and the electron acceleration mechanism with unprecedented spatial and temporal resolution.

Spectral filtering was also used to further investigate the effect of the probe-beam's spectral bandwidth and pulse duration on the temporal resolution of the shadowgrams. Using a non-polarizing beamsplitter (NPB) in the imaging system, two shadowgrams of the same plasma interaction could be recorded from the same laser shot on two cameras. An interference filter (IF) was placed either before the laser-plasma interaction, or on one of the two cameras (i.e., after the interaction). In the former case, the added positive dispersion from the filter glass was compensated using the previously described glass wedges. This was performed in order to ensure that in all cases the shortest possible pulse duration at the point of laser-plasma interaction relative to the available spectral bandwidth was used.

Figure 5 shows results from three separate laser shots. Comparing Fig. 5(b) (with an 800/40 IF placed after the interaction) to Fig. 5(a) shows a slight loss in plasma wave visibility, but the fine structures of the plasma wave remain intact. Likewise, Fig. 5(d) (with IF) shows a further loss in visibility in comparison to Fig. 5(c) (without IF) due to even narrower spectral filtering, but the temporal resolution still remains. The temporal resolution of this probing method thus proves to be independent from post-interaction spectral filtering. On the contrary, in all cases where an IF was placed before the laser-plasma interaction-thus severely lengthening the probe-beam's duration before probing the plasmathe resulting shadowgrams do not show any fine structure 
Post-Interaction Filtering: 800/40
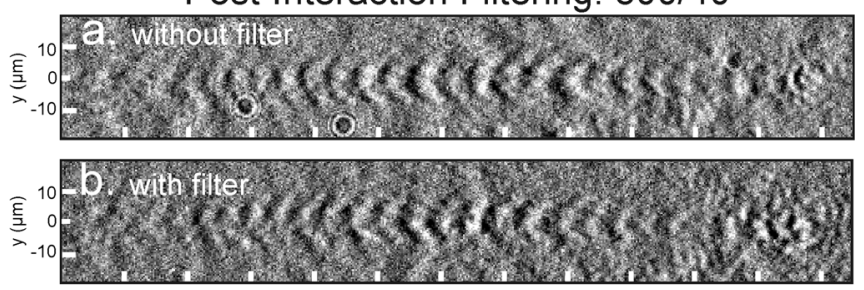

Post-Interaction Filtering: 700/10
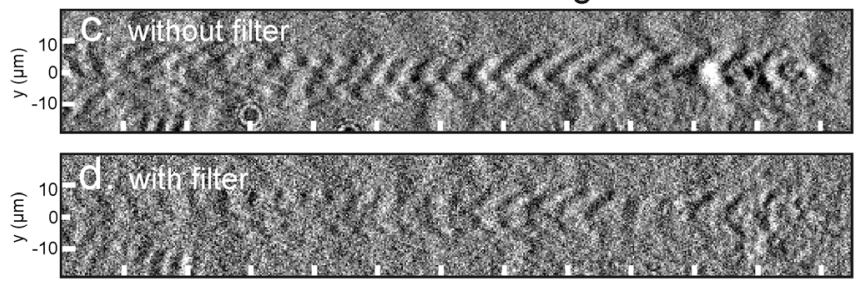

Pre-Interaction Filtering: 800/40

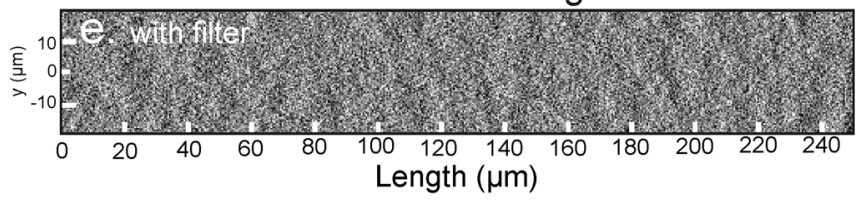

FIG. 5. Contrast-enhanced shadowgrams with and without spectral filtering. Pump-pulse propagates from left to right. Filters labeled with CW/FWHM where CW is the center wavelength $(\mathrm{nm})$ and FWHM $(\mathrm{nm})$. The image pairs ((a), (b)) and ((c), (d)) and image (e) were recorded with three separate laser shots. Comparing ((a), (b)) to ((c), (d)), the diminishing visibility of the plasma wave is a result of the probe's decreasing spectral intensity due to the narrow spectral transmission of the filters. (e) Spectral filtering before the interaction results in no discernible plasma wave structure in the shadowgram.

resembling a plasma wave (see Fig. 5(e)). The electron density modulation of the plasma wave is encoded into the spatial intensity profile of the probe-beam via refraction through the plasma wave's refractive index gradients. Because of the refractive index's relatively weak dependence on wavelength and electron density in underdense plasma, the temporal resolution of the shadowgram depends primarily on the temporal extent of the probe pulse at the location of the plasma wave, while the visibility of the images depend on the probe's spectral intensity at the CCD sensor.

While the apparent dependence between the probepulse's duration at the point of laser-plasma interaction and the shadowgram's temporal resolution is not surprising, the independence of the shadowgram's temporal resolution to post-interaction spectral filtering has some advantages. Monochromatic shadowgrams can be recorded with high temporal resolution. These images can also have higher spatial resolution due to a reduction in chromatic imaging aberrations and suppressed emitted radiation from the plasma channel. This may be advantageous for the future probing of setups with multiple diagnostic channels, as used, e.g., for the investigation of magnetic field generation in a plasma wave $^{8}$ where a few-cycle temporal resolution may offer unprecedented insight into the interaction.

In conclusion, a single-shot, few-cycle optical probe with a FWHM pulse duration of $5.9 \pm 0.4$ fs synchronized to its pump-beam was developed, characterized, and successfully implemented on the JETI $30 \mathrm{TW}$ laser system. Using an argon-filled hollow core fiber and several chirped mirrors, the probe's spectrum was broadened via self-phase modulation and temporally compressed to a pulse duration nearly one-sixth of the pump's duration used in the experiments described above. Such a probe system should be easy to implement on a large number of similar laser systems that are currently operational worldwide. Several probing geometries and imaging techniques are available to ascertain detailed information about laser-plasma interactions in underdense plasmas including the underdense regions of laser interactions involving solid targets. ${ }^{6}$ Experimental results yielding high-resolution images of a laser-driven plasma wave in the context of laser-electron acceleration highlight the large potential of this probe-beam setup. Further investigation of these interactions can help define the necessary conditions for stable relativistic electron or ion acceleration, characterize instabilities within the plasma, and potentially shed light on novel laser-plasma phenomena.

Research enabling these results received partial funding from the DFG (TR18 and KA 2869/2-1), from the European Commission's 7th Framework Program (LASERLABEUROPE, Grant No. 228334), from the Thuringian ministry for education, science, and culture through EFRE (Contract No. B715-08006), and from the BMBF (Contract No. 05K10SJ2). We gratefully acknowledge valuable contributions from B. Beleites, F. Ronneberger, and T. Rathje.

${ }^{1}$ J. Faure, Y. Glinec, A. Pukhov, S. Kiselev, S. Gordienko, E. Lefebvre, J. P. Rousseau, F. Burgy, and V. Malka, Nature 431, 541 (2004).

${ }^{2}$ S. P. D. Mangles, C. D. Murphy, Z. Najmudin, A. G. R. Thomas, J. L. Collier, A. E. Dangor, E. J. Divall, P. S. Foster, J. G. Gallacher, C. J. Hooker, D. A. Jaroszynski, A. J. Langley, W. B. Mori, P. A. Norreys, F. S. Tsung, R. Viskup, B. R. Walton, and K. Krushelnick, Nature 431, 535 (2004).

${ }^{3}$ C. G. R. Geddes, J. Van Tilborg, E. Esarey, C. B. Schroeder, D. Bruhwiler, C. Nieter, J. Cary, and W. P. Leemans, Nature 431, 538 (2004).

${ }^{4}$ A. Rousse, K. Phuoc, R. Shah, A. Pukhov, E. Lefebvre, V. Malka, S. Kiselev, F. Burgy, J.-P. Rousseau, D. Umstadter, and D. Hulin, Phys. Rev. Lett. 93, 135005 (2004).

${ }^{5}$ A. Buck, M. Nicolai, K. Schmid, C. M. S. Sears, A. Sävert, J. M. Mikhailova, F. Krausz, M. C. Kaluza, and L. Veisz, Nat. Phys. 7, 543 (2011).

${ }^{6}$ O. Jäckel, J. Polz, S. M. Pfotenhauer, H.-P. Schlenvoigt, H. Schwoerer, and M. C. Kaluza, New J. Phys. 12, 103027 (2010).

${ }^{7}$ M. C. Kaluza, M. I. K. Santala, J. Schreiber, G. D. Tsakiris, and K. J. Witte, Appl. Phys. B 92, 475 (2008).

${ }^{8}$ M. Kaluza, H.-P. Schlenvoigt, S. Mangles, A. Thomas, A. Dangor, H. Schwoerer, W. Mori, Z. Najmudin, and K. Krushelnick, Phys. Rev. Lett. 105, 115002 (2010).

${ }^{9}$ N. H. Matlis, S. Reed, S. S. Bulanov, V. Chvykov, G. Kalintchenko, T. Matsuoka, P. Rousseau, V. Yanovsky, A. Maksimchuk, S. Kalmykov, G. Shvets, and M. C. Downer, Nat. Phys. 2, 749 (2006).

${ }^{10}$ P. Dong, S. A. Reed, S. A. Yi, S. Kalmykov, G. Shvets, M. C. Downer, N. H. Matlis, W. P. Leemans, C. McGuffey, S. S. Bulanov, V. Chvykov, G. Kalintchenko, K. Krushelnick, A. Maksimchuk, T. Matsuoka, A. G. R. Thomas, and V. Yanovsky, Phys. Rev. Lett. 104, 134801 (2010).

${ }^{11}$ T. Tajima. and J. M. Dawson, Phys. Rev. Lett. 43, 267 (1979).

${ }^{12}$ D. Strickland and G. Mourou, Opt. Commun. 56, 219 (1985).

${ }^{13}$ M. Nisoli, S. De Silvestri, and O. Svelto, Appl. Phys. Lett. 68, 2793 (1996).

${ }^{14}$ S. Sartania, Z. Cheng, M. Lenzner, G. Tempea, C. Spielmann, F. Krausz, and K. Ferencz, Opt. Lett. 22, 1562 (1997).

${ }^{15}$ S. Bohman, A. Suda, T. Kanai, S. Yamaguchi, and K. Midorikawa, Opt. Lett. 35, 1887 (2010).

${ }^{16}$ A. L. Cavalieri, E. Goulielmakis, B. Horvath, W. Helml, M. Schultze, M. Fieß, V. Pervak, L. Veisz, V. S. Yakovlev, M. Uiberacker, A. Apolonski, F. Krausz, and R. Kienberger, New J. Phys. 9, 242 (2007). 
${ }^{17}$ A. Pukhov and J. Meyer-ter-Vehn, Appl. Phys. B 74, 355 (2002).

${ }^{18}$ M. Nisoli, S. Stagira, S. De Silvestri, O. Svelto, S. Sartania, Z. Cheng, G. Tempea, C. Spielmann, and F. Krausz, IEEE J. Sel. Top. Quantum Electron. 4, 414 (1998).
${ }^{19}$ G. P. Agrawal, Nonlinear Fiber Optics, 3rd ed. (Academic Press, San Diego, CA, 2001).

${ }^{20}$ R. H. Stolen and C. Lin, Phys. Rev. A 17, 1448 (1978).

${ }^{21}$ B. Landgraf, M. Schnell, A. Sävert, M. C. Kaluza, and C. Spielmann, Rev. Sci. Instrum. 82, 083106 (2011). 